\title{
Exploration on Knowledge Management Based the Cognitive Schema and its Transformation in Psychological Counseling
}

\author{
Ling Yan \\ The College of Post and Telecommunication of WIT, \\ Wuhan, China \\ Email: Yanling59@126.com
}

\begin{abstract}
In Psychological Counseling, the transformation process of the cognitive schema of the knowledge management has been receiving considerable attention in the human-system research community. And it is one of the key issues on what the cognitive structure (schema) is and how it transfers. From different perspectives, such as the cognitive science, the concepts, elements and characteristics of cognitive schema, the knowledge structure, knowledge acquisition and influence of schema on it, this paper explores the cognitive schema of knowledge management when the Counseling.
\end{abstract}

Keywords- knowledge management, cognitive schema, Psychological Counseling

In Psychological Counseling, the transformation process of the cognitive schema of the knowledge management (KM) has been receiving considerable attention in the human-system research community. And it is one of the key issues on what the cognitive structure (schema) is and how it transfers. Therefore, in practice, knowledge management often encompasses, many aspects, such us identifying and mapping intellectual assets, generating new knowledge for competitive advantage, making vast amounts of corporate information accessible. The knowledge management on the effectiveness of psychological counseling is a process which draws from a wide range of disciplines and technologies. Insights from how we learn and know will certainly improve tools and techniques for gathering and transferring knowledge, among this process, cognitive science is the core element in cognitive psychology. So, it is necessary to study the cognitive schema and its transformation in the cognitive process and thus to obtain the nearer insight into KM when the Counseling.

\section{COGNITIVE SCIENCE}

From human's Cognitive development, as an important role in the process of exploring the thinking, cognitive science can be reflected in the strategy ${ }^{[6]}$. The association areas of the cortex - those linked with thinking, memory, and language - are the last areas to develop. As they do, the child's mental abilities surge ahead ${ }^{[7]}$. Brain and mind, neural hardware and cognitive software develop together, with which, cognition can be related to all the mental activities associated with thinking, knowing, and remembering ${ }^{[4]}$. It is a product of human individual intrinsic psychological activities and refers to the operations and abilities that make the main body acquire knowledge and solve problems. The mistake cognitive science can

\author{
Wen-Ming Tian \\ The College of Post and Telecommunication of WIT, \\ Wuhan, China \\ Email: 624452444@qq.com
}

obviously lead to the wrong behavior, and ever lead to mental disorders. Much great progress has been made in all of these fields by adopting a broad perspective that places them within a more general study of the mind.

So cognitive science is concerned with the study of how organisms (especially humans) acquire, represent, manipulate, and use information. Also cognitive science is an important theoretical basis for artificial intelligence and plays a fundamental role in its development. The process is clearly related to the activity of brain. The other is, in the narrow sense, one's past knowledge and experience, which will inevitably affect and decide the choice and acceptance of later knowledge. Therefore, cognitive schema is a core issue for study and development of human in the consulting.

\section{COGNITIVE SCHEMA}

The researchers have given many names to the cognitive schema. Arising from cognitive psychology, many different terms are used to describe it: "Gestalt", "cognitive map", "plans", "reappearance representation system," "the structure", "past experience", "models", and so on. But cognitive schema has gradually become a unified description of all different fields.

No matter what the schema is called, its concept is from the same origin, and the schema theory is developed increasingly. The notion of schema as a mental representation, can be traced back to Kant's Critique of Pure Reason (1787), the origin of modern schema theory is usually identified in the work of the psychologist Bartlett, whose seminal book Remembering appeared in 1932. Bartlett found evidence that perception, understanding and memory are shaped by the expectations that people form on the basis of their prior knowledge. He used the term 'schema' to refer to the basic unit in the organization of prior knowledge. Defined as generalized knowledge about the sequence of events in particular socio-cultural contexts, schemas are mental molds into which we pour our experience ${ }^{[4]}$. Their information are stored in one's long-term memory and can be quickly recalled in the course of processing information [2]. Besides schema creation, Piaget proposed two concepts to explain how we use and adjust our schemas (1930). First, we interpret our experience in terms of our current understandings. In his terms, we use our schemas to incorporate, or assimilate, new experiences. But we also adjust, or accommodate, our 
schemas to fit the particulars of new experiences. Correspondingly, knowledge is the subject of the cognitive schema of mind. Therefore, it is necessary to discuss knowledge.

\section{KNOWLEDGE}

On many occasions knowledge has been called information. In practice, the terms information and knowledge are often used interchangeably by psychological researchers. Let's cite a typical simple working definition and get on with it: Knowledge has two basic definitions of interest. The first pertains to a defined body of information. And it also refers to a person's state of being with respect to some body of information. Besides, Gene Bellinger et al. make a thoughtful distinction in "Data, Information, Knowledge, and Wisdom". Nevertheless, Nickols provides a good, sensible, functional definition, and it is sufficient for our purposes. Nickols' two kinds of knowledge parallel Michael Polanyi's often-quoted distinction between explicit knowledge, which can be articulated in language and transferred among individuals, and tacit knowledge (also, informal knowledge), personal knowledge rooted in individual experience and involving personal belief, perspective, and values. That is just hitting human's concision of mind. When those above cannot be working normally, how could the human to get the right guidance or the right knowledge/information. It doesn't matter whether a written procedure or a subject matter expert provides a solution to a particular problem, as long as a positive result is achieved. Knowledge and information have become the medium in which management problems occur ${ }^{[3]}$. In problem solving, certainly, cognitive schema of knowledge has a greater value. However, observing how knowledge is acquired and how we can use knowledge whether tacit or explicit - in order to achieve a positive result that meets business requirements ... that is a different and very important issue in the consulting. And insights from how the cognitive schema works and how we learn and know will certainly improve tools and techniques for gathering and transferring knowledge.

\section{THE INFLUENCE OF COGNITIVE SCHEMA ON KNOWLEDGE ACQUISITION}

Knowledge acquisition is a cognitive process of human brain, and human cognitive activities are accomplished by means of human's choosing, integrating and understanding the knowledge ${ }^{[1]}$. Therefore, cognitive schema influences knowledge acquisition from the above three aspects (As Figure 1 shows). Certainly, these three aspects are related closely to each other.

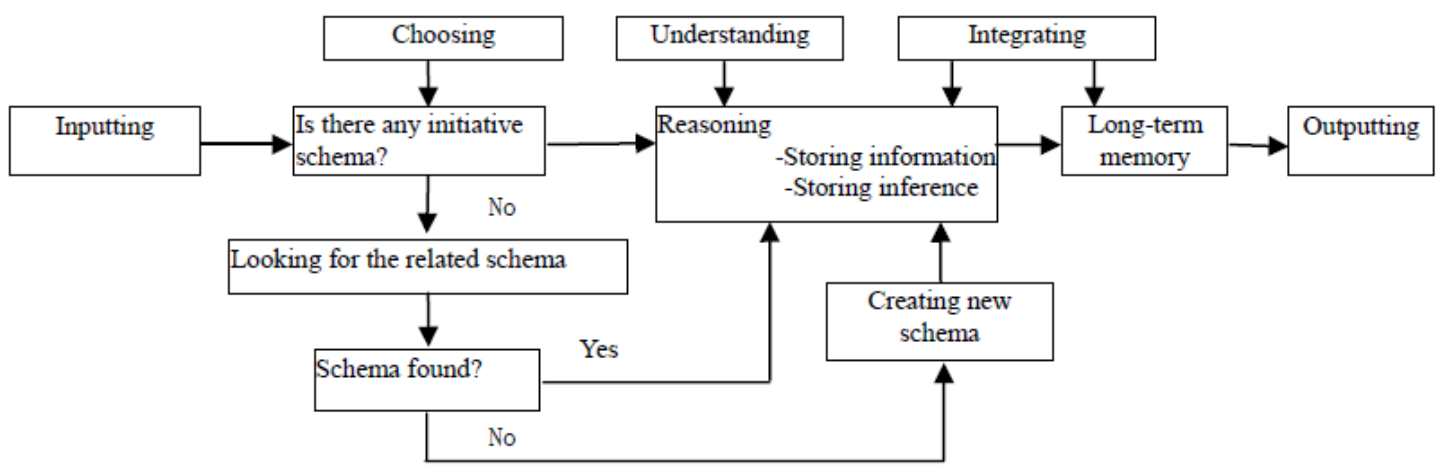

Figure1. The influence of learning on cognitive schema

\section{A. The Influence on Choosing}

In the knowledge processing system, the existing knowledge and schema play a decisive role in human behaviors and their awareness of the cognition. Take perception for example: the process of detecting a stimulus and assigning meaning to it is called perception ${ }^{[1]}$. People prefer to understand the perception based on studies conducted in Germany by the psychologists called Gestalt (another name of schema) theorists. Instead of perceiving bits and pieces of unrelated information, people perceive the whole organized meanings. The process begins with the knowledge inputting, and then tends to look for the related schema. In the perception process, the related schema accepts the external inputting and thus is activated, which makes people create internal perceptual expectations guiding the sense organs to search for knowledge purposefully.
Moreover, the cognitive schema can also be regarded as a kind of strainer. When humans try to know the objects, the objective knowledge will inevitably receive the screening of the cognitive schema. The knowledge which conforms to the main body's cognitive schema will be accepted, and that which does not will be rejected or repelled. Under the same or different kind of knowledge, schema plays an appropriate and effective role. This will shorten the responding reaction time.

In addition, people also can create a new schema when failing to find the related one and can store it for the next inputting. The process of choosing is also carrying on in the process of understanding and integrating.

\section{B. The Influence on Integrating and Understanding}

Before understanding and integrating, the inputted knowledge is often fragmented. In a mess, its 
reorganization process, to some degree, depends on the schema. Which is not a pure reorganization process but a process in which the main body draws the internal knowledge stored in his brain and uses it to deal with the external knowledge, because of the intervention of cognitive schema. Only by comparing, analyzing and synthesizing the objective knowledge through the schema can humans gain an intrinsic understanding of the objects. The process is a transformation of knowledge and schema, in which people inherit the tendency to adapt to their environment. As a person's thinking processes become more organized and new schemas develop, behavior also becomes more sophisticated and better adapted to the environment [1]. Two basic processes are involved in the transformation: assimilation and acclimation.

When clients use their existing schemas to make sense of events in the world, assimilation takes place. It involves trying to understanding something new by fitting it into what they already know (looking for the related schema). At times, they may have to distort the new information to make it fit. Just like when meeting someone, clients try to match the new experience with an existing schema for identifying the faces. In fact, with different schema, the conclusions of the matching are obviously different. According to the existing schema, people give the corresponding meaning to the related event.

But when people meet a stranger, such as clients encountering a strange idea in the consulting, what should they do? In this case as Figure 2, people must change existing schemas to respond to a new situation. If the data cannot be made to fit any existing schemas, then more appropriate structures must be developed (creating a new schema). Clients adjust thinking to fit the new knowledge, instead of adjusting the knowledge to fit their thinking. They demonstrate acclimation when they add the schema for recognizing strangers to their other systems for identifying the faces. This is acclimation. Of course, after the acclimation, they not only create a new schema but also store it through reasoning and integrating. The new schema has been put into a long-term memory and prepared for the next related inputting as an existing schema.

People adapt to the more and more complex knowledge environment by using existing schema whenever these schemas work (assimilation) or by modifying and adding to their schemas when something new is needed (acclimation). In fact, both processes are required most of the time. Even using an established pattern such as sucking through a straw may require some acclimation if the straw is of a different size of length than the type one is used to. Whenever new experiences are assimilated into an existing schema, the schema is more or less enlarged or changed, so assimilation involves some acclimation.

But sometimes neither assimilation nor acclimation is used. If people encounter something that is too unfamiliar, they may ignore it, as is mentioned above. That is to say, experience is filtered to fit the kind of thinking that a person is doing at a given time. If not, the schema will refuse the new experience.

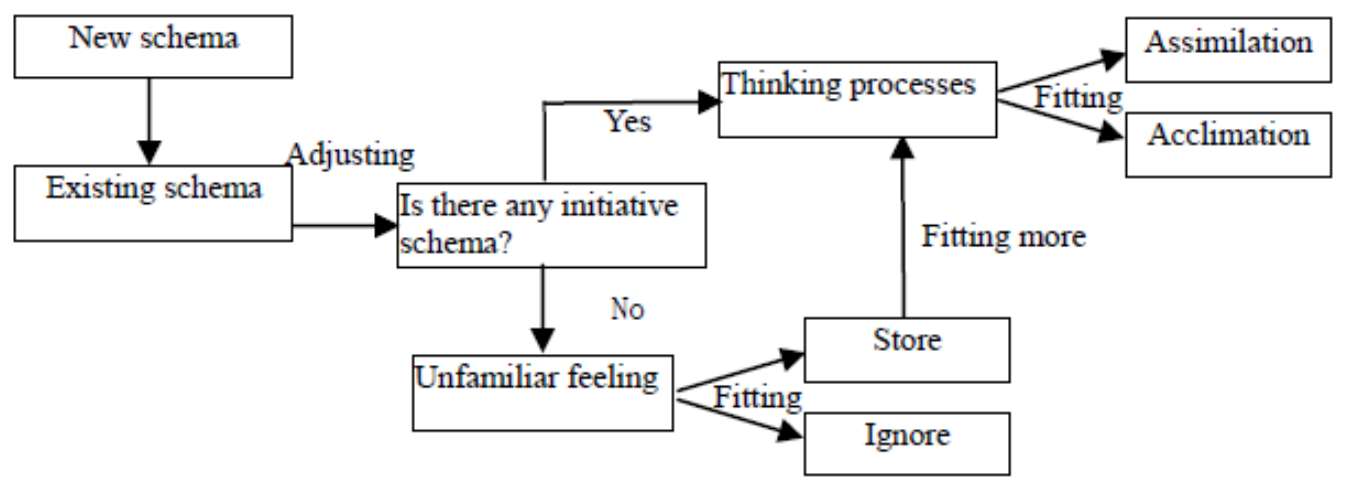

Figure2. The influence on integrating and understanding in consulting

\section{V.CONCLUSION}

From what has been discussed above, the conclusion can be drawn that schema is extensively used in human environment [7], especially in cognitive science. How to Use effective information input to cause a possible shift of cognitive schema transformation is not only of KM, but also the subject of the cognitive psychology when consulting, especially in the consulting off the practical application. Investigating the positive effects of cognitive schema based on previous theories and researches may improve learning by helping consulters identify, clarify, and organize the abstract concepts and the relationships between information being processed by visually presenting them in an almost tangible format; may elaborate and organize the information being processed to ensure that schema modification and creation are easier and happen in an organized fashion; may help consulters process the information effectively through dual coding, which uses both verbal and nonverbal (or graphic) representations to strengthen the cues to the specific information being processed and increases the possibility of information recall [5]. No matter the short-term or the long-term, cognitive schema in memory can cause the behavior change. A small effort may help the consulting processing on, so that the final consultation effectiveness will reflect. 


\section{REFERENCES}

[1] Anita Woolfolk. Education Psychology. Pearson education north Asia limited and higher education press. (2003).p. 29, 245-246.

[2] Bussmann H. Routledge Dictionary of Language and Linguistics. Tr. \& ed. G. P. Trauth, K. Kazzazi. (1995). p. 416.

[3] C. Despres, D. Chauvel. Mastering Information Management, Part 6, Knowledge Management. Financial Times. (1999). March 8, p. 4-6.

[4] David G. Myers. Psychology (Fourth Edition). New York: Von Hoffmann Press. (1995). p. 88
[5] Paivio, A. Mental representation: A dual coding approach. New York: Oxford University Press. 1986.

[6] Thatcher, R. W., Walker, R. A., \& Giudice, S. Human cerebral hemispheres develop at different rates and ages. Science, (1987). 236, 1110-11163. p.88, 145 .

[7] Wang, C. X. The instructional effects of prior knowledge and three concept mapping strategies in facilitation achievement of different educational objectives. Pennsylvania State University. 2003. 